Published online 2017 April 13.

Abstract

\title{
MRCP Findings of Hepatobiliary Fascioliasis : A Case Report
}

\author{
Shaghayegh Sadat Khabbaz, ${ }^{1, *}$ \\ ${ }^{1}$ Department of Radiology, Shahid Beheshti University of Medical Sciences (SBMU) \\ "Corresponding author: Shaghayegh Sadat Khabbaz. E-mail: shaghayegh_khabbaz@yahoo.com
}

Received 2016 December 21; Accepted 2017 February 08.

\begin{abstract}
Background: Fascioliasis, caused by Fasciola hepatica and Fasciola gigantica, is a parasitic infection. It merges in Iran as a serious problem in past decades. A pattern of fascioliasis transmission was defined for northern part of Iran, named "Caspian Pattern".

Case Presentation: We report a 33-year-old from iran who presented with epigastric and RUQ pain. We present US and MRCP findings of hepatobiliary fascioliasis and the results of her surgery. In the sonographic study, an echogenic area with no acoustic shadowing was seen in the gallblader. CBD and CHD (common hepatic duct) was slightly dilated In the MRCP elliptical intermediate signal area in $\mathrm{T} 2 \mathrm{~W}$ and iso tense area in T1W in common hepatic duct with dilated internal biliary ducts and common bile duct was seen.

Conclusions: Hepatobiliary fascioliasis is a rare disease. Imaging studies can help us for diagnosis of fascioliasis with subtle symptoms and laboratory findings especially in endemic areas.
\end{abstract}

Keywords: Fascioliasis, Magnetic Resonance Cholangiopancreatography, Ultrasonograph

This is an abstract presented in the 33rd Iranian congress of radiology (ICR) and the 15th congress of Iranian radiographic science association (IRSA). 